Bulletin of the Section of Logic

Volume 45:3/4 (2016), pp. 199-211

http://dx.doi.org/10.18778/0138-0680.45.3.4.05

Bogdan Staruch and Bożena Staruch

\title{
DECOMPOSITION OF CONGRUENCE MODULAR ALGEBRAS INTO ATOMIC, ATOMLESS LOCALLY UNIFORM AND ANTI-UNIFORM PARTS
}

\begin{abstract}
We describe here a special subdirect decomposition of algebras with modular congruence lattice. Such a decomposition (called a star-decomposition) is based on the properties of the congruence lattices of algebras. We consider four properties of lattices: atomic, atomless, locally uniform and anti-uniform. In effect, we describe a star-decomposition of a given algebra with modular congruence lattice into two or three parts associated to these properties.

Keywords: universal algebra, algebraic lattice, congruence lattice, atomic lattice, modular lattice, uniform lattice, subdirect product, star-product, decomposition of algebra

\section{Introduction}

In this paper we study properties of modular algebraic lattices in order to obtain a $\circledast$-decomposition of algebras into factors with specific congruence properties. The famous Grätzer-Schmidt theorem states that every algebraic lattice is the congruence lattice of an algebra, so we study algebraic lattices having in mind congruence lattices. In [8] there was introduced a notion of a $\circledast$-product of algebras as a special kind of a subdirect product. In the same paper, a notion of dimension of algebras was given and, in the case of algebras with modular congruence lattices, the description of a $\circledast$-decomposition of algebras into one-dimensional factors (i.e. algebras with their congruence lattices being uniform) and the one (if exists) $0^{+}$-factor (i.e. algebra with its congruence lattice being anti-uniform) was described.
\end{abstract}


Modular lattices are very interesting because many algebraic structures (groups, rings, modules, vector spaces, lattices, Boolean algebras) have modular congruence lattices.

We consider four properties of elements in lattices, namely, being an atom, uniform element, uniform atomless element and anti-uniform element. In Section 2 we recall some useful notions and facts of universal algebra and lattice theory and we give also useful notions and facts concerning $\circledast$-decomposition (from [8]). Section 3 contains technical results for modular algebraic lattices which are used in formulation and proving main results of this paper, i.e. $\circledast$-decomposition theorems, in the last section.

\section{Preliminaries}

An algebra $\mathbf{A}$ of type $F$ is an ordered pair $(A, F)$, where $A$ is a nonempty set and $F$ is a family of finitary operations on $A$.

A binary relation $\theta$ on $A$ is called a congruence on an algebra $\mathbf{A}$ of type $F$ if it is an equivalence relation on $A$ satisfying the compatibility property i.e. for each $n$-ary operation $f \in F$ and elements $a_{i}, b_{i} \in A$, if $\left(a_{i}, b_{i}\right) \in \theta$ holds for $i=1, \ldots, n$ then $\left(f\left(a_{1}, \ldots, a_{n}\right), f\left(b_{1}, \ldots, b_{n}\right)\right) \in \theta$. The set of all congruences on an algebra $\mathbf{A}$ is denoted by $\operatorname{Con} \mathbf{A}$. It is known that $\operatorname{Con} \mathbf{A}$ ordered by inclusion is an algebraic lattice which is called the congruence lattice of $\mathbf{A}$. The least element 0 is the identity relation and the greatest element 1 is $A \times A$. According to terminology like 'congruence modular algebra' and 'congruence distributive algebra', we will say that an algebra is a congruence 'Property' algebra if its congruence lattice has the property 'Property'.

An algebra $\mathbf{A}$ is a subdirect product of a family $\left(\mathbf{A}_{i}\right)_{i \in I}$ of algebras if $\mathbf{A}$ is a subalgebra of the product $\prod\left(\mathbf{A}_{i}\right)_{i \in I}$ and the projection maps $\pi_{i}: \mathbf{A} \rightarrow \mathbf{A}_{i}$ are epimorphisms for each $i \in I$.

We use two important properties of congruences. The first one states that if $\theta=\bigcap\left\{\theta_{i}\right\}_{i \in I}$, then $\mathbf{A} / \theta$ is a subdirect product of the algebras $\left(\mathbf{A} / \theta_{i}\right)_{i \in I}$. Hence if $0=\bigcap\left\{\theta_{i}\right\}_{i \in I}$, then $\mathbf{A}$ is a subdirect product of the quotient algebras $\left(\mathbf{A} / \theta_{i}\right)_{i \in I}$. The second one is The Correspondence Theorem which states that for any algebra $\mathbf{A}$ and $\theta \in \operatorname{Con} \mathbf{A}$ the interval $[\theta, 1]$ is isomorphic to $\operatorname{Con} \mathbf{A} / \theta$.

We assume throughout the paper, that $L$ is an algebraic lattice with the least element denoted by 0 and the greatest element denoted by 1 , and where $0 \neq 1$. 
This yields that if such an $L$ is a congruence lattice of an algebra $\mathbf{A}$, then $\mathbf{A}$ is nontrivial, i.e. it has at least two elements. If $L$ is algebraic then it is complete by definition. In complete lattices we have $\bigvee \emptyset=0, \wedge \emptyset=1$, $\bigvee L=1, \bigwedge L=0$.

For facts not recalled here see [1], [3], [8].

\subsection{Omitting relation, uniform and anti-uniform elements}

The relation of omitting elements is a kind of complementation. We say that an element $y \in L$ omits $x \in L$ iff $y \wedge x=0$. An element $y \in L$ omits a nonempty subset $X \subseteq L$ iff $y$ omits every element $x \in X$. If $X=\emptyset$ then every element $y \in L$ omits $X$.

For any subset $X \subseteq L$ there exists a maximal element omitting $X$. The set of all maximal elements omitting $X$ is denoted by $\mathfrak{M}_{X}$ and elements of this set are denoted by $M_{X}$. If $X=\{x\}$ then the set of all maximal elements omitting $x$ is denoted by $\mathfrak{M}_{x}$ and $M_{x}$ denotes any element of $\mathfrak{M}_{x}$.

We consider also maximal elements in $L$ omitting a given element $M_{X}$ using notation $M_{X}^{\circledast}$ for any element of the set $\mathfrak{M}_{M_{X}}$. Exceptionally, if $X=$ $\{x\}$ then for a given $M_{x}, M_{x}^{\circledast}$ denotes any element of the set $\mathfrak{M}_{x}^{\circledast}\left(M_{x}\right)=$ $\left\{z \in \mathfrak{M}_{M_{X}}: x \leq z\right\}$. Consequently, for given $M_{X}$ and $M_{X}^{\circledast} \in \mathfrak{M}_{M_{X}}$, the symbol $M_{X}^{\circledast \circledast}$ denotes any element of the set $\mathfrak{M}_{M_{X}}^{\circledast}\left(M_{X}^{\circledast}\right)$.

For example, look at the lattice on Fig.1. Here $\mathfrak{M}_{a}=\mathfrak{M}_{\alpha \wedge a}=$ $\{b, c\}, M_{a}^{\circledast}=M_{\alpha \wedge a}^{\circledast}=a$ and $M_{\{a, b\}}=c, M_{\{a, b\}}^{\circledast} \in\{a, b\}$. It is shown in [8] that in algebraic modular lattices, for any fixed $M_{X}, M_{X}=M_{X}^{\circledast \circledast}$ independently of the choice of $M_{X}^{\circledast}$. Moreover, $M_{X}$ is uniquely determined for any $X$ in algebraic distributive lattices.

We recall here the definition of a uniform element in a lattice, known from papers of Grzeszczuk and Puczyłowski [4], [5]. An element $u \in L \backslash\{0\}$ is uniform in $L$ if $x \wedge y \neq 0$ for any $x, y \in(0, u]$. A lattice $L$ is uniform if 1 is uniform.

The set of all uniform elements in $L$ will be denoted by $U(L)$. The set of all atoms of $L$ will be denoted by $A(L)$. Every atom is uniform, so $A(L) \subseteq U(L)$. A lattice $L$ is atomic if for any $x \in L \backslash\{0\}$ there is an atom $a \in L$ such that $a \leq x$. A lattice $L$ is atomless if $A(L)=\emptyset$. A uniform element $u$ will be called atomless, if the interval $[0, u]$ is an atomless lattice. The set of all atomless uniform elements in $L$ will be denoted by $N(L)$, and obviously, $N(L) \subseteq U(L)$. Thus $A(L) \cap N(L)=\emptyset$ and $A(L) \cup N(L) \subseteq U(L)$.

A lattice $L$ is locally uniform if for any $x \in L \backslash\{0\}$ there exists a $u \leq x$ such that $u \in U(L)$. 
Every chain lattice is uniform. The lattice of all subgroups of the group of integers $Z$ is atomless uniform. The lattice of all subgroups of the group $Z \times Z$ is locally uniform, but not uniform. Generally, the lattice of all subgroups of the power $Z^{n}, n \geq 2$, is locally uniform and there are infinitely many uniform elements in $Z^{n}$.

An element $t \in L \backslash\{0\}$ is anti-uniform in $L$ iff $U([0, t])=\emptyset$. The set of all anti-uniform elements in $L$ is denoted by $T(L)$. By definition every anti-uniform element in $L$ omits $U(L)$, thus $U(L) \cap T(L)=\emptyset$. A lattice $L$ is anti-uniform if 1 is anti-uniform. The Lindenbaum-Tarski algebra with an infinite set of variables is an anti-uniform lattice.

An element $e \in L$ is called essential in $L$ if $x \wedge e \neq 0$ for every $x \in L \backslash\{0\}$. The 1 is essential in $L$. If an $x$ is essential in $L$ then $M_{x}=0$. We say that an element $e \in L$ is essential in an element $a \in L$ iff $e \leq a$ and $e$ is essential in the interval $[0, a] \subseteq L$.

\subsection{Modular lattices}

A lattice $L$ is modular iff for any $x, y, z \in L$, if $z \leq x$ then $x \wedge(y \vee z)=(x \wedge y) \vee z$.

Recall some properties of modular lattices.

M1 A lattice $L$ is modular iff it satisfies the shearing identity: $x \wedge(y \vee z)=x \wedge((y \wedge(x \vee z)) \vee z)$

M2 A lattice $L$ is modular iff it satisfies the identity: $(x \vee y) \wedge(x \vee z)=x \vee(y \wedge(x \vee z))$

M3 For any modular lattices $L$ the Isomorphism Theorem holds, i.e. for any $a, b \in L$ the intervals $[a, a \vee b]$ and $[a \wedge b, b]$ are isomorphic and the isomorphism is $\varphi_{b}(x)=x \wedge b$, while the inverse isomorphism is $\psi_{a}(x)=x \vee a$.

We give below two facts (see [8]) useful in our proofs.

FM1 Let $L$ be a modular lattice. Let $a, b, c \in L \backslash\{0\}$ be such that $a \wedge b=$ $a \wedge c=b \wedge c=0$ and let $\alpha=(a \vee b) \wedge(c \vee b)>b$. Then $\alpha \wedge a \neq 0$ and $\alpha \wedge c \neq 0$ and the intervals $[0, \alpha \wedge a],[b, \alpha],[0, \alpha \wedge c]$ are isomorphic (see Fig. 1.).

FM2 Let $L$ be a modular lattice. Then

1. $x \vee M_{x}$ is essential in $L$ for any $x, M_{x} \in L$,

2. if $\emptyset \neq X \subseteq L$ then $M_{X} \vee M_{X}^{\circledast}$ is essential in $L$ for any $M_{X}, M_{X}^{\circledast}$. 


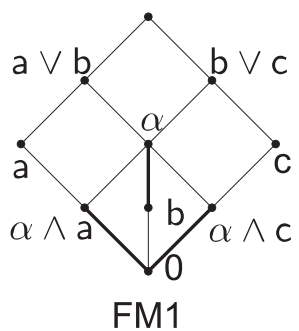

Fig. 1.

\subsection{Independent sets, star-sets and star-products}

The idea of introducing independent sets has its origin in linearly independent sets of vectors. There are several definitions of independent sets in lattices (see e.g. [3], [6], [9], [5]). We say that a subset $X \subseteq L \backslash\{0\}$ is independent if $|X|=1$, or $|X|>1$ and for every $x \in X$ it holds that $x \wedge \bigvee(X \backslash\{x\})=0$

We will use notation $\bar{y}=\bigwedge(Y \backslash\{y\})$ and $\bar{Y}=\{\bar{y}: y \in Y\}$.

For any independent set $X=\left\{x_{i}\right\}_{i \in I}$, we use the symbol $M_{i}^{X}$ to denote any maximal element omitting $x_{i}$ such that $\bigvee\left(X \backslash\left\{x_{i}\right\}\right) \leq M_{i}^{X}$.

DEFINITION 2.1. [8]

1. Let $X, Y \subseteq L$ and $X=\left\{x_{i}\right\}_{i \in I}, Y=\left\{y_{i}\right\}_{i \in I}$. We say that the pair $(X, Y)$ is a star pair (a $\circledast$-pair) in $L$ iff

(a) $X$ is an independent set in $L$ such that $\bigvee X$ is essential in $L$,

(b) $\bigwedge Y=0$,

(c) $y_{i}$ is a maximal element omitting $x_{i}$ such that $\bigvee\left(X \backslash\left\{x_{i}\right\}\right) \leq y_{i}$ is essential in $y_{i}$, for every $i \in I$.

2. We say that $Y=\left\{y_{i}\right\}_{i \in I}$ is a star-set (a $\circledast$-set) in $L$ iff $(\bar{Y}, Y)$ is a $\circledast$-pair in $L$.

3. An algebra $\mathbf{A}$ is a star-product (a $\circledast$-product) of algebras $\left(\mathbf{A}_{i}\right)_{i \in I}$ if $\mathbf{A}$ is a subdirect product of this family such that the set $Y=\left\{k e r \pi_{i}\right\}_{i \in I}$ of congruences on $\mathbf{A}$ is a $\circledast$-set. If $\mathbf{A}$ is a $\circledast$-product of algebras $\left(\mathbf{A}_{i}\right)_{i \in I}$ we use notation $\circledast\left(\mathbf{A}_{i}\right)_{i \in I}$. 
Directly from definition we have that for any algebra $\mathbf{A}$, if $Y=\left\{\theta_{i}\right\}_{i \in I}$ is a $\circledast$-set in $C o n \mathbf{A}$ then $\mathbf{A} \simeq \circledast\left(\mathbf{A} / \theta_{i}\right)_{i \in I}$.

It is worth mentioning here that every $\circledast$-set $Y$ is irredundant, that is, $\bigwedge Y=0$ and $\bigwedge(Y \backslash\{y\})=\bar{y} \neq 0$ for any $y \in Y$. Moreover, if $|Y|>1$ then $y \neq 0$ and $y$ is not essential in $L$ for every $y \in Y$.

TheOrem 2.2. [8] Let $L$ be a modular lattice. Then

1. if $X$ is an independent set in $L$ and $y \wedge \bigvee X=0$ for some $y \in L \backslash\{0\}$, then $\{y\} \cup X$ is independent in $L$,

2. $X$ is a maximal independent set in $L$ iff $X$ is an independent set and $\bigvee X$ is essential in $L$,

3. If $X$ is a maximal independent set in $L$ such that $|X|>1$, then $\left\{M_{x}^{X}\right\}_{x \in X}$ is $a \circledast$-set, and if $L=$ Con $\mathbf{A}$ then $\mathbf{A} \simeq \circledast\left(\mathbf{A} / M_{x}^{X}\right)_{x \in X}$,

4. $\left\{M_{X}, M_{X}^{\circledast}\right\}$ is a $\circledast$-set in $L$ for any $\emptyset \neq X \subseteq L$. So, if $L=C$ Con $\mathbf{A}$ then $\mathbf{A} \simeq \mathbf{A} / M_{X} \circledast \mathbf{A} / M_{X}^{\circledast}$.

\section{Four kinds of elements and their properties in modular lattices}

We assume in this section that $L$ is an algebraic modular lattice. We consider here four properties of elements in $L$, namely, belonging to one of the sets $A(L), N(L), T(L)$ or $U(L)$. If there is no confusion, we omit the symbol of the lattice $L$ in notation of omitting elements, for example, we write $M_{A}$ instead of $M_{A(L)}$ and $M_{A}^{\circledast}$ instead of $M_{A(L)}^{\circledast}$ etc.

The following two facts follow directly from definition:

FACT 3.1. For any $x \in L$

1. if $x \in U(L)$ then $x \notin T(L)$,

2. if $x \in T(L)$ then $x \notin P(L)$ for any $P \in\{A, N, U\}$,

3. if $x \in A(L)$ then $x \notin P(L)$ for any $P \in\{N, T\}$,

4. if $x \in N(L)$ then $x \notin P(L)$ for any $P \in\{A, T\}$.

FACT 3.2. Let $P, Q \in\{A, N, T\}, P \neq Q$ and $x \in P(L)$.

1. If $x^{\prime} \in L \backslash\{0\}$ is such that $x^{\prime} \leq x$ then $x^{\prime} \in P(L)$.

2. if $y \in Q(L)$ then $x \wedge y=0$.

The following fact is a simple consequence of Fact 3.2. 
FACT 3.3. For any $P \in\{A, N, T\}$ and for any $y \in L \backslash\{0\}$

1. if $x \in P([0, y])$ then $x \in P(L)$,

2. if $x \in P(L)$ and $x \wedge y>0$, then $x \wedge y \in P([0, y])$.

Proposition 3.4. For any $x \in L \backslash\{0\}$ there exists an $x^{\prime} \in L \backslash\{0\}$ with $x^{\prime} \leq x$ such that

1. $x^{\prime}$ belongs to exactly one of the sets $U(L), T(L)$,

2. $x^{\prime}$ belongs to exactly one of the sets $A(L), N(L), T(L)$,

3. $M_{U} \wedge M_{T}=0$ for any $M_{U}, M_{T}$,

4. $M_{A} \wedge M_{N} \wedge M_{T}=0$ for any $M_{A}, M_{N}, M_{T}$.

Proof. Let $x \in L \backslash\{0\}$. If $x \in T(L)$ then by definition $x^{\prime} \notin U(L)$ for every $x^{\prime} \leq x$. If $x \notin T(L)$ then there is a $u \in U(L)$ such that $u \leq x$. If $u \in N(L)$ then $u \notin A(L)$. If $u \notin N(L)$ then there is an $a \in A(L)$ such that $a \leq u \leq x$. For the proof of (iii) assume that $0 \neq x \in M_{U} \wedge M_{T}$. Then $x \in M_{U}$ omits all uniform elements, so, by definition, $x \in T(L)$ and hence $x \wedge M_{T}=0$. A contradiction. The proof of (iv) is analogous.

To simplify formulation and proofs of some results we use the following terminology:

Let $\{P, Q, R\}=\{A, N, T\}$. We say that a lattice $L$ :

- is satisfying $P$ iff $P(L) \neq \emptyset$ and $Q(L)=\emptyset=R(L)$,

- is satisfying $\{P, Q\}$ iff $P(L) \neq \emptyset \neq Q(L)$ and $R(L)=\emptyset$.

The next fact shows how some properties of lattices are expressed in this terminology:

FACT 3.5. $L$ is satisfying:

1. $A$ iff $L$ is atomic,

2. $N$ iff $L$ is atomless locally uniform,

3. $T$ iff $L$ is anti-uniform,

4. $\{A, N\}$ then $L$ is locally uniform,

5. $\{N, T\}$ then $L$ is atomless,

6. $\{A, T\}$ then $L$ has no atomless uniform element.

Proof. The proof, based on Proposition 3.4, is very simple. We show (i) as example. If $L$ is satisfying $A$ then $A(L) \neq \emptyset$ and $T(L)=\emptyset=N(L)$. Then for any $x \in L \backslash\{0\}$ there is an atom $a \leq x$ and thus $L$ is atomic. If 
$L$ is atomic then for any $x \in L \backslash\{0\}$ there exists an atom $a \leq x$. Hence $A(L) \neq \emptyset$ and $T(L)=\emptyset=N(L)$ and thus $L$ is satisfying $A$.

FACT 3.6. For any $P \in\{A, N, T\}$ and any $x \in L \backslash\{0\}$, if $L$ is satisfying $P$ then $[0, x]$ is satisfying $P$.

It is worth mentioning here that the four properties, that we describe in this paper, are preserved by isomorphism. We point out here a useful fact concerning isomorphisms and intervals.

FACT 3.7. Let $a, b, c, d \in L$ and $a<b, c<d$. Assume that $[a, b] \simeq[c, d]$, where $\phi:[a, b] \rightarrow[c, d]$ is the isomorphism. Let $P, Q \in\{A, N, T\}, P \neq Q$. Then

1. $p \in P([a, b])$ iff $\phi(p) \in P([c, d])$,

2. $[a, b]$ is satisfying $P$ iff $[c, d]$ is satisfying $P$,

3. $[a, b]$ is satisfying $\{P, Q\}$ iff $[c, d]$ is satisfying $\{P, Q\}$.

Lemma 3.8. Let $P, Q \in\{A, N, T\}, P \neq Q$. Then for any $M_{P}, M_{P}^{\circledast}$ the following holds:

1. $M_{P} \wedge q>0$ for any $q \in Q(L)$,

2. $M_{P}^{\circledast} \wedge p>0$ for any $p \in P(L)$,

3. $M_{P}^{\circledast} \wedge q=0$ for any $q \in Q(L)$.

Proof. (i) Let $q \in Q(L)$. If $M_{P} \wedge q=0$ then $M_{P} \vee q>M_{P}$. By maximality of $M_{P}$ there exists a $p \in P(L)$ such that $p \leq M_{P} \vee q$. Use FM1, where $a=q, b=M_{P}, c=p$. Then $\alpha=\left(M_{P} \vee q\right) \wedge\left(M_{P} \vee p\right)=M_{P} \vee p>M_{P}$. Then $\alpha \wedge p=\left(M_{P} \vee p\right) \wedge p=p$ and $\alpha \wedge q=\left(M_{P} \vee p\right) \wedge q=q^{\prime} \leq q$ and thus $[0, p] \simeq\left[0, q^{\prime}\right]$, which contradicts Fact 3.2 since $p \in P(L)$ and $q^{\prime} \leq q \notin P(L)$. (ii) Let $p \in P(L)$ and assume that $M_{P}^{\circledast} \wedge p=0$. Then $M_{P}^{\circledast} \vee p>M_{P}^{\circledast}$ and hence $M_{P} \wedge\left(M_{P}^{\circledast} \vee p\right)>0$. Notice also that $p \wedge\left(M_{P} \wedge\left(M_{P}^{\circledast} \vee p\right)\right) \leq$ $p \wedge M_{P}=0$. Now use FM1, where $a=M_{P}, b=p, c=M_{P}^{\circledast}$. Then by M2, $\alpha=\left(M_{P} \vee p\right) \wedge\left(M_{P}^{\circledast} \vee p\right)=p \vee\left(M_{P} \wedge\left(M_{P}^{\circledast} \vee p\right)\right)>p$. Hence there is an isomorphism $\phi:\left[0, M_{P} \wedge \alpha\right] \rightarrow\left[0, M_{P}^{\circledast} \wedge \alpha\right]$ and $M_{P} \wedge \alpha>0$. Thus there exists an $x \in\left[0, M_{P} \wedge \alpha\right]$ and $R \in\{A, N, T\} \backslash\{P\}$ such that $x \in R(L)$ according to Proposition 3.4. Thus $\phi(x) \in\left[0, M_{P}^{\circledast} \wedge \alpha\right] \cap R(L)$. In view of (i) we have $\phi(x) \wedge M_{P}>0$. Therefore $M_{P}^{\circledast} \wedge M_{P}>0$, a contradiction.

(iii) Let $q \in Q(L)$. If $q \wedge M_{P}^{\circledast}=q^{\prime}>0$ then $q^{\prime} \wedge M_{P}=0$ and hence $q^{\prime} \vee M_{P}>M_{P}$. By maximality of $M_{P}$ there exists a $p \in P(L)$ such that $p \leq q^{\prime} \vee M_{P}$. Thus, using M1 we get $0<p^{\prime}=M_{P}^{\circledast} \wedge p \leq M_{P}^{\circledast} \wedge\left(M_{P} \vee q^{\prime}\right)=$ 
$M_{P}^{\circledast} \wedge\left(\left(M_{P} \wedge\left(M_{P}^{\circledast} \vee q^{\prime}\right)\right) \vee q^{\prime}\right)=M_{P}^{\circledast} \wedge\left(\left(M_{P} \wedge M_{P}^{\circledast}\right) \vee q^{\prime}\right)=M_{P}^{\circledast} \wedge q^{\prime}=q^{\prime}$. Hence $p^{\prime} \leq q^{\prime}$, but $p^{\prime} \leq p \in P(L)$ and $q^{\prime} \leq q \notin P(L)$. A contradiction.

Lemma 3.9. Let $\{P, Q, R\}=\{A, N, T\}$. Then

1. for any $M_{P}, M_{P}^{\circledast}$ there exist $M_{Q}, M_{R}$ such that $M_{P}^{\circledast}=M_{Q} \wedge M_{R}$,

2. if $L$ is satisfying $\{P, Q\}$ then $\left\{M_{P}, M_{Q}\right\}$ is a $\circledast$-set in $L$,

3. if $A(L), N(L), T(L)$ are all nonempty then for any $M_{T}$ there exist $M_{A}, M_{N}$ such that $\left\{M_{T}, M_{A}, M_{N}\right\}$ is a $\circledast$-set in $L$.

Proof. (i) If $P(L)=\emptyset$ then $M_{P}=1$ and $M_{P}^{\circledast}=0$, and by Proposition 3.4, $0=M_{P} \wedge M_{Q} \wedge M_{R}=M_{Q} \wedge M_{R}$. Let $A(L), N(L), T(L)$ be all nonempty. By Lemma $3.8 M_{P}^{\circledast}$ omits $Q(L) \cup R(L)$, so there are $M_{Q} \geq M_{P}^{\circledast}$ and $M_{R} \geq M_{P}^{\circledast}$. As $M_{Q} \wedge M_{R}$ omits $M_{P}$ and $M_{P}^{\circledast} \leq M_{Q} \wedge M_{R}$, by maximality of $M_{P}^{\circledast}$ we get $M_{P}^{\circledast}=M_{Q} \wedge M_{R}$.

(ii) follows from (i), since $M_{R}=1$ and then $M_{P}^{\circledast}=M_{Q}$. Due to Theorem $2.2\left\{M_{P}, M_{Q}\right\}$ is a $\circledast$-set in $L$.

(iii) Let us take $M_{T}, M_{T}^{\circledast}$ and choose $M_{A}, M_{N}$ as in (i).

Let $Y=\left\{M_{T}, M_{A}, M_{N}\right\}$. First we show that that $\bar{Y}$ is a maximal independent set in $L$. Recall that (see page 203) $\bar{Y}=\left\{\bar{M}_{T}, \bar{M}_{A}, \bar{M}_{N}\right\}$, where $\bar{M}_{T}=M_{A} \wedge M_{N}=M_{T}^{\circledast}, \bar{M}_{A}=M_{N} \wedge M_{T}, \bar{M}_{N}=M_{A} \wedge M_{T}$.

It is easy to see that $\bar{M}_{A} \wedge \bar{M}_{N}=0$. Moreover,

$\bar{M}_{T} \wedge\left(\bar{M}_{A} \vee \bar{M}_{N}\right)=M_{T}^{\circledast} \wedge\left(\left(M_{T} \wedge M_{N}\right) \vee\left(M_{T} \wedge M_{A}\right)\right) \leq M_{T}^{\circledast} \wedge M_{T}=0$. Due to Theorem 2.2 (i) $\bar{Y}$ is an independent set.

Now, we show that $\bigvee \bar{Y}$ is essential in $L$. Let $x \in L \backslash\{0\}$, then by Proposition 3.4 there exists an $x^{\prime}$ such that $0<x^{\prime} \leq x$ and $x^{\prime}$ belongs to exactly one of the sets $T(L), A(L), N(L)$.

If $x^{\prime} \in T(L)$ then $0<x^{\prime} \wedge M_{T}^{\circledast}=x^{\prime} \wedge \bar{M}_{T}$.

If $x^{\prime} \in A(L)$ then $0<x^{\prime} \wedge M_{T} \in A(L)$ then $0<\left(x^{\prime} \wedge M_{T}\right) \wedge M_{N}=x^{\prime} \wedge \bar{M}_{A}$. If $x^{\prime} \in N(L)$ then $0<x^{\prime} \wedge M_{T} \in N(L)$ then $0<\left(x^{\prime} \wedge M_{T}\right) \wedge M_{A}=x^{\prime} \wedge \bar{M}_{N}$.

Thus $x \wedge \bigvee \bar{Y} \neq 0$. Hence by Theorem 2.2, $\bar{Y}$ is a maximal independent set. Thus, to prove that $Y$ is a $\circledast$-set, we have to show that for any $P \in\{A, N, T\}, M_{P}=M_{\bar{M}_{P}}$. So, let $\{P, Q, R\}=\{A, N, T\}$. Then $M_{P} \geq$ $\bar{M}_{Q} \vee \bar{M}_{R}=\left(M_{P} \wedge M_{Q}\right) \vee\left(M_{P} \wedge M_{R}\right)$, and $M_{P}$ omits $\bar{M}_{P}$. Hence $M_{P} \leq$ $M_{\bar{M}_{P}}^{\bar{Y}}$, for any $P \in\{A, N, T\}$. Obviously, $M_{T}=M_{\bar{M}_{T}}^{\bar{Y}}=M_{U}^{\circledast}$, so we will show this equality for $M_{A}$ and $M_{N}$. 
If $M_{A}<x \leq M_{\bar{M}_{A}}$, then there exists an $a \in A(L)$ such that $a<x$ and $a \wedge M_{N}>0$ and $a \wedge M_{T}>0$. Thus

$0<a=\left(a \wedge M_{N}\right) \wedge\left(a \wedge M_{T}\right)=a \wedge\left(M_{N} \wedge M_{T}\right) \leq M_{\bar{M}_{A}} \wedge \bar{M}_{A}=0$. This yields a contradiction. Similarly, if $M_{N}<x \leq M_{\bar{M}_{N}}$, then there exists $n \in N(L)$ such that $n<x$ and $n \wedge M_{A}>0$ and $n \wedge M_{T}>0$. Thus $0<\left(n \wedge M_{A}\right) \wedge\left(n \wedge M_{T}\right)=n \wedge\left(M_{A} \wedge M_{T}\right) \leq M_{\bar{M}_{N}} \wedge \bar{M}_{N}=0$.

Finally, $\left\{M_{T}, M_{A}, M_{N}\right\}$ is a $\circledast$-set in $L$.

Lemma 3.10. For any $P \in\{A, N, T\}$ and $M_{P}, M_{P}^{\circledast}$

1. if $A(L), N(L), T(L)$ are all nonempty then

(a) $\left[0, M_{P}\right]$ and $\left[M_{P}^{\circledast}, 1\right]$ are satisfying $\{Q, R\}=\{A, N, T\} \backslash\{P\}$,

(b) $\left[M_{P}, 1\right]$ and $\left[0, M_{P}^{\circledast}\right]$ are satisfying $P$,

2. if $L$ is satisfying $\{P, Q\}$, then

(a) $\left[0, M_{P}\right]$ and $\left[M_{P}^{\circledast}, 1\right]$ are satisfying $Q$,

(b) $\left[M_{P}, 1\right]$ and $\left[0, M_{P}^{\circledast}\right]$ are satisfying $P$.

Proof. (i) (a) If $x \in P\left(\left[0, M_{P}\right]\right)$ then $x \in P(L)$, but $M_{P}$ omits $P(L)$, so $P\left(\left[0, M_{P}\right]\right)=\emptyset$. Let $\{Q, R\}=\{A, N, T\} \backslash\{P\}$. If $x \in Q(L)$ or $x \in R(L)$ then $0<x \wedge M_{P} \in Q(L)$ or $0<x \wedge M_{P} \in R(L)$. Hence $\left[0, M_{P}\right]$ is satisfying $\{Q, R\}$. Then by the Isomorphism Theorem $\left[M_{P}^{\circledast}, M_{P} \vee M_{P}^{\circledast}\right]$ is satisfying $\{Q, R\}$. Thus $Q\left(\left[M_{P}^{\circledast}, 1\right]\right) \neq \emptyset$ and $R\left(\left[M_{P}^{\circledast}, 1\right]\right) \neq \emptyset$, and $P\left(\left[M_{P}^{\circledast}, M_{P} \vee M_{P}^{\circledast}\right]\right)=\emptyset$.

If $x \in P\left(\left[M_{P}^{\circledast}, 1\right]\right)$, then $M_{P}^{\circledast}<x^{\prime}=\left(x \wedge M_{P}\right) \vee M_{P}^{\circledast} \leq M_{P} \vee M_{P}^{\circledast}$, and $x^{\prime} \leq x$, thus $x^{\prime} \in P\left(\left[M_{P}^{\circledast}, M_{P} \vee M_{P}^{\circledast}\right]\right)=\emptyset$. A contradiction. Other statements have analogous proofs.

There are some interesting consequences of the last proposition, because we obtain some 'bottom' parts of the lattice, which have specific properties, and similarly, 'top' parts. The 'bottom' parts have a crucial role in finding bases and dimensions of lattices (see [8]). The 'top' parts are used in $\circledast$-decompositions of algebras. For example, if $L$ is locally uniform not atomic nor atomless, then $L$ has a 'bottom' atomic part $\left[0, M_{N}\right]$ and a 'bottom' atomless part $\left[0, M_{A}\right]$ and has a 'top' atomic part $\left[M_{A}, 1\right]$ and a 'top' atomless part $\left[M_{N}, 1\right]$. 


\section{Star-decomposition of congruence modular algebras}

We apply the results of the last section to congruence lattices of congruence modular algebras. The following proposition, which describes $\circledast$-decomposition of algebras into congruence atomic, congruence atomless locally uniform and congruence anti-uniform parts, is a straightforward consequence of Lemmas 3.9, 3.10 and the Correspondence Theorem.

TheOrem 4.1. Let $\mathbf{A}$ be a nontrivial congruence modular algebra and $L=$ Con $\mathbf{A}$. Let $\{P, Q, R\}=\{A, N, T\}$. Then

1. if $L$ is satisfying $\{P, Q\}$ then $\mathbf{A} \simeq \mathbf{A} / M_{P} \circledast \mathbf{A} / M_{Q}$ and $\mathbf{A} / M_{P}$ is congruence satisfying $P$ and $\mathbf{A} / M_{Q}$ is congruence satisfying $Q$,

2. if $A(L), N(L), T(L)$ are all nonempty, then for any $M_{T}$ there exist $M_{A}, M_{N}$ such that $\mathbf{A} \simeq \mathbf{A} / M_{T} \circledast \mathbf{A} / M_{A} \circledast \mathbf{A} / M_{N}$, and $\mathbf{A} / M_{T}$ is congruence anti-uniform, $\mathbf{A} / M_{A}$ is congruence atomic, $\mathbf{A} / M_{N}$ is congruence atomless locally uniform.

Substituting for $P, Q$, some properties from $\{A, N, T\}$ we can obtain the following:

COROLlary 4.2. Let $\mathbf{A}$ be a nontrivial congruence modular algebra and $L=\operatorname{Con} \mathbf{A}$.

1. If $L$ is satisfying $\{A, N\}$ then $\mathbf{A}$ is congruence locally uniform and $\mathbf{A} \simeq \mathbf{A}_{1} \circledast \mathbf{A}_{2}$, where $\mathbf{A}_{1}$ is congruence atomic and $\mathbf{A}_{2}$ is congruence atomless locally uniform.

2. If $L$ is satisfying $\{N, T\}$ then $\mathbf{A}$ is congruence atomless and $\mathbf{A} \simeq \mathbf{A}_{1} \circledast \mathbf{A}_{2}$, where $\mathbf{A}_{1}$ is congruence atomless locally uniform and $\mathbf{A}_{2}$ is congruence anti-uniform.

3. If $L$ is satisfying $\{A, T\}$ then $\mathbf{A}$ has no atomless uniform element in its congruence lattice and $\mathbf{A} \simeq \mathbf{A}_{1} \circledast \mathbf{A}_{2}$, where $\mathbf{A}_{1}$ is congruence atomic and $\mathbf{A}_{2}$ is congruence anti-uniform.

4. If $A(L), N(L), T(L)$ are all nonempty, then $\mathbf{A} \simeq \mathbf{A}_{1} \circledast \mathbf{A}_{2} \circledast \mathbf{A}_{3}$, where $\mathbf{A}_{1}$ is congruence anti-uniform, $\mathbf{A}_{2}$ is congruence atomic, $\mathbf{A}_{3}$ is congruence atomless locally uniform.

The next proposition answers the question if properties of factors in a subdirect decomposition of the given algebra can be preserved in some $\circledast$-decomposition of this algebra. 
Theorem 4.3. Let $\mathbf{A}, \mathbf{B}_{1}$ and $\mathbf{B}_{2}$ be nontrivial congruence modular algebras. Assume that $\mathbf{A}$ is a subdirect product of algebras $\mathbf{B}_{1}$ and $\mathbf{B}_{2}$. Then

1. if $\mathbf{A}$ is congruence satisfying $\{P, Q\}$, and $\mathbf{B}_{1}$ is congruence satisfying $P$ and $\mathbf{B}_{2}$ is congruence satisfying $Q$, then there exist algebras $\mathbf{A}_{1}, \mathbf{A}_{2}$ such that

(a) $\mathbf{A}_{i}$ is a homomorphic image of $\mathbf{B}_{i}$ for $i=1,2$,

(b) $\mathbf{A}_{1}$ is congruence satisfying $P, \mathbf{A}_{2}$ is congruence satisfying $Q$,

(c) $\mathbf{A} \simeq \mathbf{A}_{1} \circledast \mathbf{A}_{2}$,

2. if $A(L), N(L), T(L)$ are all nonempty, and $\{P, Q, R\}=\{A, N, T\}$, and $\mathbf{B}_{1}$ is congruence satisfying $P$ and $\mathbf{B}_{2}$ is congruence satisfying $\{Q, R\}$, then there exist algebras $\mathbf{A}_{1}, \mathbf{A}_{2}$ such that

(a) $\mathbf{A}_{i}$ is a homomorphic image of $\mathbf{B}_{i}$ for $i=1,2$,

(b) $\mathbf{A}_{1}$ is congruence satisfying $P, \mathbf{A}_{2}$ is congruence satisfying $\{Q, R\}$,

(c) $\mathbf{A} \simeq \mathbf{A}_{1} \circledast \mathbf{A}_{2}$.

Proof. In both cases, let $\theta_{1}=k e r \pi_{1}, \theta_{2}=k e r \pi_{2}$, where $\pi_{i}$ is the $i$-th projection from $\mathbf{A}$ onto $\mathbf{B}_{i}$. Then $\theta_{1} \wedge \theta_{2}=0$ and $\theta_{1}, \theta_{2}<\theta_{1} \vee \theta_{2}$.

(i) By the Correspondence Theorem $\left[\theta_{1}, 1\right]$ is satisfying $P$, so $\left[\theta_{1}, \theta_{1} \vee \theta_{2}\right]$ is satisfying $P$, and thus $\left[0, \theta_{2}\right]$ is satisfying $P$. Similarly, $\left[\theta_{2}, 1\right]$ is satisfying $Q$, hence $P\left(\left[\theta_{2}, 1\right]\right)=\emptyset$. Then $P\left(\left[\theta_{2}, \theta_{1} \vee \theta_{2}\right]\right)=P\left(\left[0, \theta_{1}\right]\right)=\emptyset$. Thus $\theta_{1}$ omits $P(L)$, so $\theta_{1} \leq M_{P}$ for some $M_{P}$. On the other side $\theta_{2}$ omits $M_{P}$, so $\theta_{2} \leq M_{P}^{\circledast}$ for some $M_{P}^{\circledast}$. Moreover, $\left[M_{P}, 1\right]$ is satisfying $P,\left[M_{P}^{\circledast}, 1\right]$ is satisfying $Q$ and $\left\{M_{P}, M_{P}^{\circledast}\right\}$ is a $\circledast$-set in $L$. Hence $\mathbf{A}_{1}=\mathbf{A} / M_{P}$ is a homomorphic image of $\mathbf{B}_{1}$ and $\mathbf{A}_{2}=\mathbf{A} / M_{P}^{\circledast}$ is a homomorphic image of $\mathbf{B}_{2}$ and $\mathbf{A} \simeq \mathbf{A}_{1} \circledast \mathbf{A}_{2}$.

(ii) Analogously, there exist $M_{P}, M_{P}^{\circledast}$ such that $\theta_{1} \leq M_{P}, \theta_{2} \leq M_{P}^{\circledast}$, and moreover, $\left[M_{P}, 1\right]$ is satisfying $P,\left[M_{P}^{\circledast}, 1\right]$ is satisfying $\{Q, R\}$ and $\left\{M_{P}, M_{P}^{\circledast}\right\}$ is a $\circledast$-set in $L$. Hence $\mathbf{A}_{1}=\mathbf{A} / M_{P}$ is a homomorphic image of $\mathbf{B}_{1}$ and $\mathbf{A}_{2}=\mathbf{A} / M_{P}^{\circledast}$ is a homomorphic image of $\mathbf{B}_{2}$ and $\mathbf{A} \simeq \mathbf{A}_{1} \circledast \mathbf{A}_{2}$.

Notice that $M_{P}, M_{P}^{\circledast}$ are uniquely determined in distributive algebraic lattices, so all the $\circledast$-decompositions described in this section are uniquely determined for congruence distributive algebras e.g. lattices and Boolean algebras. 


\section{References}

[1] S. Burris, H. P. Sankappanavar, A Course in Universal Algebra, Springer-Verlag, (1981).

[2] A. W. Goldie, The structure of prime rings under ascending chain conditions, Proc. London Math. Soc. (3), 8 (1958), pp. 589-608.

[3] G. Grätzer, General Lattice Theory. Second edition. Birkhauser Verlag, Basel (1998).

[4] P. Grzeszczuk, E. R. Puczyłowski, On Goldie and dual Goldie dimensions, J. Pure Appl. Algebra 31 (1984), pp. 47-54.

[5] P. Grzeszczuk, E. R. Puczyłowski, On infinite Goldie dimension of modular lattices and modules, J. Pure Appl. Algebra 35 (1985), pp. 151-155.

[6] J. Krempa, On lattices, modules and groups with many uniform elements, Algebra Discrete Math., 1 (2004), pp. 75-86.

[7] E. R. Puczyłowski, A linear property of Goldie dimension of modules and modular lattices, J. Pure Appl. Algebra 215 (2011), pp. 1596-1605.

[8] B. Staruch, Irredundant decomposition of algebras into one-dimensional factors, submitted to Bulletin of the Section of Logic (2016).

[9] A. P. Zolotarev, On balanced lattices and Goldie dimension of balanced lattices, Siberian Math. J., 35:3 (1994), pp. 539-546.

Department of Mathematics and Computer Science University of Warmia and Mazury, Olsztyn, Poland bstar@uwm.edu.pl,bostar@matman.uwm.edu.pl 